\title{
Le système éducatif finlandais
}

\section{Marie-Hélène Margelidon}

\section{(2) OpenEdition}

\section{Journals}

Édition électronique

URL : http://journals.openedition.org/ries/1548

DOI : 10.4000/ries. 1548

ISSN : 2261-4265

\section{Éditeur}

Centre international d'études pédagogiques

\section{Édition imprimée}

Date de publication : 1 septembre 2004

Pagination : 175-182

ISBN : 978-2-85420-561-8

ISSN : 1254-4590

\section{Référence électronique}

Marie-Hélène Margelidon, «Le système éducatif finlandais », Revue internationale d'éducation de Sèvres [En ligne], 36 I septembre 2004, mis en ligne le 21 novembre 2011, consulté le 23 avril 2019. URL : http://journals.openedition.org/ries/1548; DOI : 10.4000/ries.1548

Ce document a été généré automatiquement le 23 avril 2019

(c) Tous droits réservés 


\title{
Le système éducatif finlandais
}

\author{
Marie-Hélène Margelidon
}

1 Dans chaque pays, le système éducatif est enraciné dans une réalité historique, politique et sociale. En Finlande, quatre éléments majeurs sont à prendre en considération :

\section{Sur le plan historique}

2 Dans la Finlande annexée par la Suède au XII ${ }^{e}$ siècle, l'enseignement est entièrement sous la dépendance de l'Église. En 1809, la Finlande devient grand duché de l'empire russe mais la réglementation scolaire russe n'y est pas introduite. La direction nationale des écoles, créée en 1869, met fin à la domination sans partage de l'Église sur l'éducation. En 1917, la Finlande accède enfin à l'indépendance et, dans la constitution adoptée en 1919, deux principes sont affirmés: la généralisation de l'obligation scolaire généralisée et la gratuité de l'enseignement primaire. La loi sur la scolarité obligatoire est promulguée en 1921 et rapidement mise en œuvre. C'est en 1970 qu'intervient une réforme essentielle qui consiste à combiner l'école primaire et le collège en une seule école, "l'école fondamentale », qui s'adresse à tous et qui définit la scolarité obligatoire.

3 Sur le plan linguistique, la Finlande est officiellement bilingue et ses deux langues officielles sont le finnois et le suédois depuis 1862. Ces deux langues étant peu parlées à l'extérieur du pays et la population étant peu nombreuse, la pratique des langues étrangères s'est naturellement imposée aux Finlandais. Lors de la création de l'école fondamentale, il a été décidé que chaque élève apprendrait, en plus de la deuxième langue du pays, une langue étrangère obligatoire qui pourrait être l'anglais, l'allemand, le français ou le russe. Cette offre a été préservée, la Suède voisine servant de contreexemple, même si, en pratique, c'est l'anglais qui domine. Dès cette époque, la Finlande s'est engagée sur la voie de la diversification des langues, largement soutenue en cela par son opinion publique. Le pays a de fait vingt ans d'avance sur les objectifs européens en la matière. 


\section{Sur le plan social}

4 La Finlande est animée par un souci d'égalité qui s'inscrit dans une longue tradition propre aux pays nordiques. Il s'agit de "donner aux élèves des chances égales d'enseignement et [d']aplanir les obstacles à l'apprentissage, en particulier en ce qui concerne les élèves en difficulté $»^{1}$. Ces principes sont essentiels dans la politique éducative de la Finlande depuis le début du XX⿳亠丷厂 siècle. Cette tradition s'articule autour de deux axes : faible différenciation entre les écoles (la plus faible parmi les pays de l'OCDE) et similitude des cursus et des programmes dans toutes les écoles et lycées du pays. L'enquête PISA (Programme international pour le suivi des acquis des élèves) révèle que cette uniformité est en fait un des facteurs clés propices à un taux élevé de réussite ${ }^{2}$. En Finlande, même l'école dont les résultats sont les moins bons parvient à un taux de compétence en lecture plus élevé que celui des autres écoles des pays de l'OCDE. Des chercheurs finlandais ${ }^{3}$, avancent comme cause principale de ce succès «la variation moyenne entre les résultats des élèves parmi les plus faibles des pays de l'OCDE, un écart entre les résultas les plus faibles et les meilleurs relativement faible ». Il faut noter que les relativement bonnes compétences des élèves en difficulté expliquent le taux moyen aussi élevé des compétences en lecture. L'institution développe les recherches, en particulier au niveau de l'enseignement fondamental, sur les stratégies facilitatrices d'éveil à la lecture qui atténuent les inégalités dues au contexte socio-économique. Il semble que la flexibilité des cursus scolaires et le choix des matières enseignées laissé aux élèves soient des éléments essentiels pour la réussite.

\section{Sur le plan politique}

5 La Finlande est entrée dans l'Union européenne en 1995. Comme le souligne le ministère de l'Éducation nationale finlandaise, "l'action et l'influence de la Finlande exigent une vision nationale claire et une hiérarchisation des objectifs; la participation aux programmes concernant la recherche, l'éducation, la culture et la jeunesse sont d'une importance cruciale $»^{4}$. Le haut niveau de formation de la population est un point essentiel de la stratégie nationale. Cet objectif implique le respect des communautés linguistiques. Ainsi l'enseignement est-il dispensé en finnois, suédois et en same (dans les parties septentrionales de la Laponie finlandaise). Il passe par une coopération entre les responsables de la formation et le monde du travail pour faire en sorte que l'offre éducative corresponde mieux aux exigences de ce dernier. Il suppose également la promotion de la mobilité via une participation active aux programmes d'échanges multilatéraux.

\section{Fonctionnement administratif du système éducatif}

\section{L'État}

6 Les principes généraux et la législation qui régissent la politique en matière d'éducation et de formation sont décidés par le Parlement. La mise en œuvre de cette politique incombe au conseil des ministres, au ministère de l'Éducation, à la direction nationale de l'Enseignement et au conseil de l'Évaluation. Au niveau de l'administration centrale, les principaux partenaires sont le ministère du Travail, des Affaires sociales et de la Santé, 
responsable des jardins d'enfants, ainsi que le ministère du Commerce et de l'Industrie, qui s'intéresse aux politiques industrielle et technologique à travers Tekes, l'agence nationale pour la technologie, et le VTT, le centre de recherche technique en Finlande. C'est le ministère de l'Éducation qui répartit les budgets et délègue aux municipalités les moyens financiers et d'enseignement en fonction des effectifs et des structures. Le ministère détermine les disciplines d'enseignement, délègue à la direction nationale de l'Enseignement la conception des programmes et de l'évaluation. En 1998, les dépenses publiques de l'éducation et de la formation représentaient $6,2 \%$ du PIB, soit un taux supérieur à celui de la moyenne de la zone OCDE.

\section{Les municipalités}

7 Les communes ont une autonomie de gestion et un droit d'imposition. La Finlande compte 452 communes qui déterminent, en fonction de leur budget, les heures d'enseignement qu'elles ajouteront aux dotations allouées par le ministère. Elles embauchent et gèrent les enseignants, construisent les établissements, assurent la maintenance et la restauration. Cette dernière est généralement centralisée et les repas sont distribués dans chaque école (et gratuits dans l'école de base).

\section{Fonctions des différents acteurs}

Un(e) Pääjohtaja (conseiller national de l'Éducation) préside l'Opetus-hallitus ou direction nationale de l'Enseignement, qui est l'administration spécifique qui répond du développement des objectifs, contenus et méthodes de formation dans l'enseignement fondamental, les lycées, l'enseignement professionnel et la formation pour adultes. Elle détermine les fondements des programmes scolaires, procède à l'évaluation du système éducatif et prépare en particulier la définition et l'évaluation du diplôme national donnant droit à l'inscription à l'université ou «matriculation». Cette direction compte trois cent fonctionnaires et spécialistes des questions éducatives et dispose d'une grande autonomie par rapport au ministère. Jusqu'en 2003, l'évaluation était assumée par la direction nationale mais, dans un souci d'objectivité et de transparence, un conseil autonome, l'Arviointineuvosto, conseil de l'Évaluation, a été créé depuis. Il organise l'évaluation avec le ministère.

\section{Organisation de la scolarité}

9 L'enseignement est normalement gratuit, quel que soit le niveau de la formation. L'enseignement fondamental l'est complètement mais, à partir du second degré, les élèves peuvent avoir à payer le matériel pédagogique, les repas et les voyages. Les élèves et les étudiants qui, après l'école fondamentale, entreprennent à plein temps des études, reçoivent un soutien financier. Toutes les études donnent droit à ce soutien qui peut en principe se prolonger jusqu'au doctorat et est même accordé pour les études à l'étranger. Ce soutien comprend une aide financière directe, une allocation de logement et la garantie de l'État pour les prêts bancaires. 


\section{Qui dispense l'enseignement?}

10 D'après la loi, une association ou une fondation peut être autorisée à dispenser un enseignement au même titre que les communes ou même l'État. C'est ce qu'on appelle en Finlande une école privée. Ces écoles doivent conclure un accord avec la commune dans laquelle elles se situent. Les écoles privées établies avant 1998 sont subventionnées par l'État à $100 \%$, les autres sont financées à hauteur de $90 \%$.

11 Les écoles privées doivent suivre normalement les mêmes cadres de programmes que tous les autres établissements et doivent soumettre leur programme d'enseignement au bureau d'enseignement de la commune. Quelques-unes suivent une philosophie pédagogique spécifique comme Montessori ou Steiner et il existe un tout petit nombre d'écoles religieuses ou d'écoles dans lesquelles la langue d'instruction est une langue étrangère.

\section{Enseignement préscolaire}

12 L'enseignement préscolaire désigne l'éducation et l'enseignement dispensés aux enfants durant l'année qui précède le début de l'instruction, à partir de sept ans. Il est facultatif mais la loi du $1^{\text {er }}$ août 2000 annonce un changement important: désormais, tous les enfants de six ans ont accès à la "classe zéro». Les grandes orientations ont été déterminées en 1996 : respect de l'individualité de l'enfant, apprentissage actif et travail en équipe sont les objectifs essentiels. Les notions qui doivent être abordées sont l'approche des mathématiques, la nature, l'environnement, la religion, la morale, la gymnastique, la santé et les arts. La majeure partie de cet enseignement est dispensée dans des jardins d'enfants qui dépendent du ministère des Affaires sociales ou dans les classes « zéro » des établissements scolaires qui dépendent du ministère de l'Éducation.

\section{L'école fondamentale}

13 L'école fondamentale dure neuf ans et s'adresse aux enfants de sept à seize ans. Il existe 3672 écoles fondamentales qui regroupent 570000 élèves, 68 écoles privées (moins de $2 \%$ ) et 244 écoles spéciales qui scolarisent 11200 élèves de moins de seize ans. Le coût par élève s'élève à 3760 euros, soit un coût de 550 euros par habitant. La loi prévoit que l'enseignement doit être mis en œuvre près du domicile de l'enfant. L'organisation des transports scolaires de plus de cinq kilomètres et les frais qu'ils entraînent sont pris en charge par l'instance qui organise l'enseignement, la municipalité dans la plupart des cas. Les enfants sont répartis par classe d'âge: pendant les six premières années, un professeur principal enseigne la plupart des matières ; durant les trois dernières années, l'enseignement se fait par discipline. L'orientation pédagogique et un enseignement spécifique, si nécessaire, sont inclus dans le cursus de l'école fondamentale. Les élèves apprennent leur langue maternelle, la deuxième langue nationale, des langues étrangères, les mathématiques, la physique, la chimie, l'histoire, l'instruction civique, l'éducation physique et sportive, la musique, les arts figuratifs, le travail manuel, la religion ou des connaissances d'éthique, la biologie et la géographie ainsi que l'environnement. À la rentrée 2004, un enseignement nouveau sera mis en place, l'éducation à la santé, sans modifier toutefois le nombre d'heures de cours par semaine, ce qui pose quelques problèmes. 
6,2 \% des élèves commencent l'apprentissage de la première langue obligatoire (A1) en première année, $12,8 \%$ en deuxième année et les autres en troisième année, le plus souvent l'anglais, le suédois, l'allemand, le français ou le russe ; en septième année, la deuxième langue obligatoire (B1) est le plus souvent le suédois ou l'anglais. Un nombre croissant d'établissements tend à introduire la première langue étrangère dès la première année. Les élèves ont la possibilité d'étudier deux autres langues étrangères (A2) et de nombreux élèves en abordent l'étude dès la cinquième année. Les statistiques montrent que les filles choisissent plus souvent une autre langue vivante étrangère que l'anglais et sont plus nombreuses à étudier une langue facultative. Elles représentent deux tiers des effectifs de français et $60 \%$ de ceux de russe ou d'allemand. En fait, ce sont les municipalités qui décident du choix des langues en fonction des effectifs. Le same (langue des Lapons) connaît trois versions qui peuvent toutes être enseignées et présentées au baccalauréat.

La fin de la scolarité à l'école fondamentale n'est pas sanctionnée par un examen; elle qualifie pour l'enseignement du second degré et les redoublements ou abandons sont extrêmement rares.

\section{L'enseignement du second degré}

16 Deux possibilités s'offrent aux élèves: l'enseignement du lycée ou l'enseignement professionnel. Les lycées sélectionnent eux-mêmes leurs élèves sur la base de leurs résultats à l'école fondamentale. Les études durent de deux à quatre ans mais le rythme d'apprentissage des connaissances et la progression dans les études sont individuels. Les études sont organisées en modules de cours et les choix fixent le rythme du travail de l'élève.

17 En raison des choix très divers des élèves, le lycée ne différencie ni les classes ni les années; le baccalauréat ou "matriculation» sanctionne les études et comprenait, jusqu'en 2004, quatre épreuves obligatoires: langue maternelle, seconde langue nationale, langue étrangère et mathématiques ou épreuve combinant l'ensemble des autres matières. À partir de 2005, le suédois ne sera plus obligatoire à la «matriculation » mais son apprentissage restera obligatoire. Outre les matières obligatoires, le candidat peut choisir des matières optionnelles. C'est la commission du baccalauréat, relevant du ministère de l'Éducation, qui fixe les épreuves et effectue la double correction, la première ayant été effectuée par les professeurs des candidats. Une réforme concernant les langues vivantes étrangères sera mise en place en 2005 , l'évaluation de la production orale intervenant en 2008. La "matriculation » est un diplôme de fin d'études. L'accès à l'université se fait sur épreuves d'admission, le nombre de places pour les nouveaux étudiants représentant environ un tiers des effectifs de la classe d'âge. En 2004, on a compté 31021 bacheliers dont 2022 suédophones.

18 L'enseignement secondaire à distance donne aussi aux élèves la possibilité d'étudier à leur propre rythme, indépendamment de leur lieu de résidence. Ils peuvent sélectionner leur cursus et déterminer leur progression.

19 Dans l'enseignement professionnel, les études durent trois ans et donnent droit à l'admission aux études supérieures. Les établissements professionnels sélectionnent les élèves, principalement sur examen de leur réussite scolaire antérieure et diverses épreuves. On dénombre 75 diplômes professionnels de base. La formation professionnelle se caractérise par des études théoriques, des périodes de travaux dirigés ainsi que des 
stages pratiques; cet enseignement est gratuit pour tous les élèves. Dans le cadre du contrat d'entreprise, l'organisateur de la formation, l'employeur et l'élève passent un contrat de travail d'une durée déterminée. En outre, le diplôme peut revêtir la forme d'un diplôme-test, au cours duquel, indépendamment du mode d'apprentissage, l'élève peut, au moyen d'une épreuve à caractère démonstratif, faire la preuve qu'il maitrise les connaissances et les aptitudes requises par sa future profession.

\section{L'enseignement supérieur}

En Finlande, c'est l'université qui a la plus longue tradition. Le premier site universitaire, l'académie royale de Turku, fut établi en 1640 puis transféré à Helsinki en 1828 sous l'appellation d'Université impériale Alexandre. Il devint ensuite l'Université d'Helsinki, la seule université jusqu'au début du XIX ${ }^{e}$ siècle au cours duquel furent créées l'université de technologie et l'école supérieure de commerce et d'administration. Dans les années 1920, deux universités furent ouvertes à Turku, l'une de langue finnoise et l'autre en suédois. L'enseignement supérieur comprend deux niveaux parallèles.

\section{Les écoles supérieures professionnelles}

21 Au nombre de vingt-neuf, mises en place dans les années 1990 pour donner une formation supérieure de type non universitaire à partir des établissements qui dispensaient un enseignement professionnel postérieur au second degré, elles accueillaient 129000 étudiants en 2003. La "matriculation" (ou un diplôme professionnel de base) est nécessaire pour l'admissibilité à l'enseignement professionnel supérieur qui est un enseignement encore jeune. Les écoles supérieures professionnelles se distinguent des universités dans la mesure où elles ne sont pas gérées par l'État mais par les communes voire par des entités privées. L'État en assume toutefois le financement à hauteur de $57 \%$. Les études durent entre trois ans et demi et quatre ans auxquels il faut ajouter des stages professionnels en entreprise.

\section{Les universités}

Leur mission est de faire progresser la recherche en dispensant l'enseignement nécessaire, mais également, et c'est le sens de " la troisième dimension » qu'une nouvelle législation en préparation veut mettre en place, d'établir une coopération avec la société civile. Les vingt universités finlandaises sont autonomes. Elles peuvent conduire des recherches en toute indépendance dans le cadre des lois et directives élaborées par le ministère de l'Éducation.

Toutes les universités sont gérées par l'État qui les finance à hauteur de $65 \%$. Chaque université conclut avec le ministère de l'Éducation un contrat de trois ans dans lequel les objectifs opérationnels sont définis.

Le nombre des étudiants a augmenté d'environ $40 \%$ au cours des dix dernières années. La Finlande compte aujourd'hui 170000 étudiants dont 21900 en troisième cycle. Les étudiants choisissent plutôt la technologie, les études littéraires et les sciences naturelles et c'est en art, théâtre, danse et sciences vétérinaires que l'on trouve les plus faibles effectifs. 17000 diplômes sont délivrés dont 12400 pour le mastère et 1200 pour les doctorats. 
flexibilité qui se traduit par la grande liberté de choix offerte à l'élève dès le secondaire égalité des chances qui se traduit, notamment via le développement de l'enseignement secondaire à distance, par une " ardente obligation » d'une qualité égale d'enseignement sur tout le territoire.

\section{BIBLIOGRAPHIE}

OCDE / Institut de statistiques de l'UNESCO : PISA 2000 : Programme international pour le suivi des acquis des élèves.

OCDE Education Committee : Review of Education Policy in Finland, 23 May 2002. 
Finnish Success in PISA-And some Reasons Behind it, Institute for Educational Research, university of Yuväskylä (Finland).

OCDE 2004 : Education and Equity.

L'Éducation et la formation en Finlande, ministère des Affaires étrangères (Finlande), 2002.

Rapport sur l'enseignement des langues en Finlande, National Board of Education, 2001.

L'Enseignement en Finlande, ministère de l'Éducation (Finlande), 1999.

Pisa : l'École en procès?, Assises Romandes de l'Éducation, Université de Lausanne, 2002.

Finlande : situation de l'enseignement et de l'apprentissage des langues étrangères, Ritta Piri, ministère de l'Éducation nationale, 2002.

Finnish Universities 2002, Ministry of Education.

University System in Finland, Jussi Tanner, Iiris Patosalmi et Markku Mattila: Ministry of Education. Strategy 2015, Ministry of Education.

Promoting Language Learning and Linguistic Diversity: An Action Plan 2004; Commission of The European Communities.

Centre pour la mobilité sociale « CIMO » : www.cimo.fi

Direction nationale de l'enseignement : www.oph.fi

\section{NOTES}

1. . L'enseignement en Finlande, Opetushallitus 1999, p. 4.

2. Programme international pour le suivi des acquis des élèves (PISA), OCDE, 2000.

3. The Finnish success in PISA - and some reasons behind it, University of Jyväskylä, 2002.

4. Ministry of Education Strategy 2015.

5. Jukka Sarjola, directeur général de la direction nationale de l'Enseignement, septembre 2002.

\section{INDEX}

Mots-clés : système éducatif

Index géographique : Finlande 